\title{
Research on Vibration Reduction of Half-Vehicle Active Suspension Sys- tem Based on Time-delayed Feedback Control with Wheel Displacement
}

\author{
Yong GUO*, Chuanbo REN** \\ *School of Mechanical Engineering, Shandong University of Technology, Zibo 255000, China, \\ E-mail: guoyong20080808@126.com \\ **School of Transportation and Vehicle Engineering, Shandong University of Technology, Zibo 255000, China, \\ E-mail: chuanbor@sdut.edu.cn (Corresponding author) \\ crossref http://dx.doi.org/10.5755/j02.mech.28809
}

\section{Introduction}

Vehicle suspension system behaves as one of the most important parts of modern automobile, and its vibration isolation performance has an important influence on the ride comfort and driving safety of vehicle. In general, the conventional passive suspension system consists of elastic components and damper with fixed parameters. Although it has the advantages of simple structure and low cost, its structural parameters cannot be automatically adjusted with the complex and changeable external conditions and different driving states of vehicles. The suspension system can achieve the optimal performance only under a specific combination of speed and road conditions. Aiming at the limitation of traditional vehicle passive suspension system in coordinating vehicle ride comfort and safety, experts and scholars at home and abroad have continuously developed various active and semi-active suspension systems with good performance on the basis of traditional passive suspension systems [1-3] in recent years. That is, according to the driving condition of the vehicle, the actuator can exert control force on the system to improve the ride comfort and safety of the vehicle. However, with the application of active and semi-active control, the suspension system will inevitably appear a series of delay factors such as signal acquisition, transmission, controller calculation and actuator delay. The existence of time delay will inevitably reduce the performance of the suspension system, and may even lead to the instability of the suspension system, thus affecting the safety of vehicle driving. Song and $\mathrm{Xu}$ [4] investigated the stochastic preview control with time-delay consideration for an active vehicle suspension system with look-ahead sensor, simulation results demonstrated that time delay should be considered in the stochastic preview control of an active suspension system, especially when large time delay exited. If the suspension system with time delay was controlled by the optimal controller designed with no consideration of time delay, instability in responses might occur and the control effects possibly got worse with preview time increase. Kou and Fan et al. [5] designed a kind of the semi-active suspension with EHA to improve the ride comfort and stability of the vehicle, which the results show that the EHA semi-active suspension has good damping controllability, However, with the increase of time delay, the "jump vibration" phenomenon happens to the suspension system. In order to improve the control accuracy and stability of suspension system, the time delay compensation technology and modern control technology are combined to reduce or eliminate the adverse effects of time delay. [6-7] However, with the in- depth study of time-delay by other scholars, it is found that time-delay can change the effective frequency band of saturation control, if the time delay is adjusted as a feedback control parameter, the vibration control effect of the main system can be effectively improved [8-9]. Olgac and HolmHansen [10] firstly introduced the time-delayed feedback control into the dynamic vibration absorber and proposed the concept of time-delay resonator. The results show that: by choosing the appropriate feedback control parameters (feedback gain, feedback delay), the delayed resonator can completely absorb the vibration of the main system under harmonic excitation, and the vibration of the main system disappears. At present, some scholars have studied the vehicle suspension control system with active time delay. Du and Zhang [11] constructed a two-degree-of-freedom vehicle active suspension system with time-delayed state feedback control. The optimal objective functions are the sprung mass acceleration, suspension dynamic stroke and tire dynamic load. The dynamic performance of suspension is discussed and the influence of time delay on suspension performance is given. Liu and Sun et al. [12] constructed a twodegree-of-freedom vehicle suspension model based on timedelayed feedback control with vertical acceleration of vehicle body, and the particle swarm optimization algorithm is adopted to study the optimization of time-delayed feedback control parameters of vehicle suspension system under different road harmonic excitation frequencies in finite frequency band. The results show that: compared with the passive suspension system, the acceleration amplitude of the vehicle body in the finite frequency band can be reduced by at least $19.6 \%$. To sum up, from the perspective of the utilization of time delay, it is rare to introduce time delay into the field of vehicle engineering applications, and the key to improve the performance of suspension system is to obtain the optimal feedback control parameters. However, the research on time-delayed feedback control of vehicle suspension system is still in the stage of basic theory, and the main problems are as followings:

1. The research on time-delayed vibration reduction control strategy and control effect of vehicle active suspension system is mostly based on the 2-DOF vehicle suspension system with single time-delayed feedback control such as vertical acceleration of vehicle body. There are few researches on the time-delayed vibration reduction strategy and control effect of vehicle active suspension system with multi-degree of freedom and multi-delayed feedback control based on other time-delayed feedback objects (such as wheel and engine).

2. Usually, only the local vibration reduction effect 
of the vertical acceleration of the vehicle body near a resonance frequency point under the optimal time-delayed feedback control is concerned in the vehicle active suspension system. There is no accurate understanding of the pitch angular acceleration, the output of suspension dynamic travel and tire dynamic displacement which affect the control performance of vehicle suspension system under optimal timedelayed feedback control.

3. Compared with the fixed form of road harmonic excitation, the vibration reduction effect of vehicle active suspension system based on time-delayed feedback control under road random excitation is less significant than that under simple harmonic excitation. In view of this, in order to deal with the problem of complex time-varying excitation such as external multi-frequency, the general calculation method for solving the appropriate time-delayed feedback control parameters still needs to be further explored.

In order to further improve the vibration reduction effect of vehicle active suspension system based on timedelayed feedback control under road random excitation, in this paper, the mechanical model of 4-DOF vehicle active suspension system based on double time-delayed feedback control is studied. The optimization strategy of equivalent harmonic excitation combined with particle swarm optimization algorithm is adopted to obtain the optimal values of time-delayed feedback control parameters (feedback gain and feedback delay) of vehicle active suspension system under the road random excitation. The time-domain simulation results show that: compared with the passive suspension system, the vehicle active suspension system based on the time-delayed feedback control with vertical displacement of wheel can significantly improve the ride comfort and stability of the vehicle under the optimal time-delayed feedback control, which provides a theoretical basis for the selection of time-delayed feedback control strategy and the optimal design of time-delayed feedback control parameters of vehicle suspension system.

\section{Mechanical model of time-delayed feedback control for vehicle active suspension system}

In order to simplify the research problem, the vehicle vibration caused by road roughness is mainly studied in this paper without considering the influence of unbalanced force generated by engine and transmission system, as well as other factors of the vehicle itself on the body vibration. The mechanical model of active suspension system of vehicle based on time-delayed feedback control is constructed on the basis of vehicle passive control suspension system with magnetorheological damper as active control actuator by referring to the vibration reduction mechanism of timedelayed dynamic vibration absorber, as shown in Fig. 1 and the design parameters of vehicle active suspension system are shown in the Table 1.

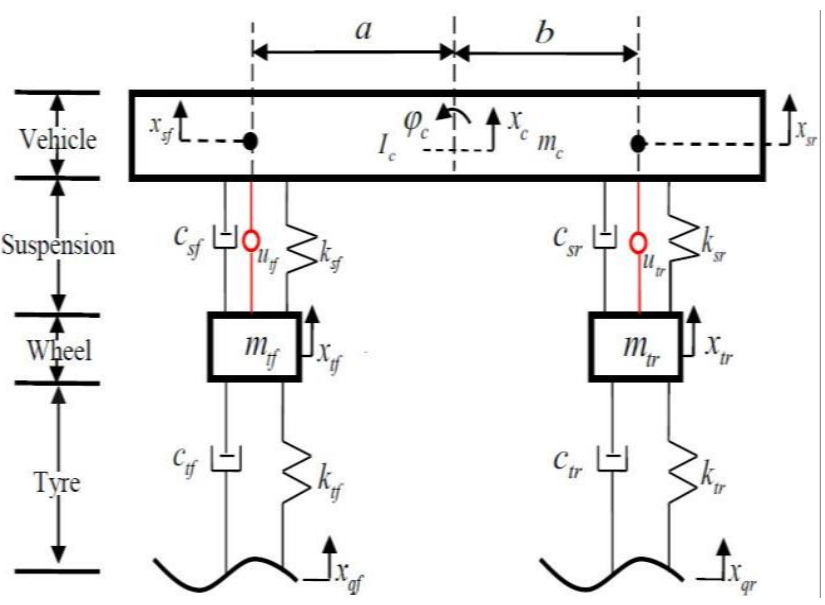

Fig. 1 Half-vehicle vibration model: where $u_{t f}$ is feedback force of the front suspension; $u_{t r}$ is feedback force of the rear suspension; $a$ is distance from the center of mass of the body to the front axle; $b$ is distance from the center of mass of the body to the rear axle; $m_{t f}$ is unsprung mass of front suspension; $m_{t r}$ is unsprung mass of rear suspension; $k_{s f}$ is equivalent stiffness of front suspension; $k_{s r}$ is equivalent stiffness of rear suspension; $c_{s f}$ is front suspension damping; $c_{s r}$ is rear suspension damping; $k_{t f}$ is tire stiffness of the front wheels; $k_{t r}$ is tire stiffness of the rear wheels; $m_{c}$ is body mass; $I_{c}$ is moment of inertia; $x_{s f}$ is vertical displacement of the sprung mass of the front suspension; $x_{s r}$ is vertical displacement of the sprung mass of the rear suspension; $x_{c}$ is vertical displacement at the mass center of the body; $\varphi_{c}$ is pitch angle at the mass center of the body; $x_{t f}$ is vertical displacement of the non-sprung mass of the front suspension; $x_{t r}$ is vertical displacement of the non-sprung mass of the rear suspension; $x_{q f}$ is road roughness of the front wheels; $x_{q r}$ is road roughness of the rear wheels

Table 1

Model parameters of vehicle suspension

\begin{tabular}{|c|c|c|c|}
\hline Parameter & Value & Parameter & Value \\
\hline$m_{c}, \mathrm{~kg}$ & 690 & $I_{c}, \mathrm{~kg} \cdot \mathrm{m}^{2}$ & 1222 \\
\hline$m_{t f}, \mathrm{~kg}$ & 40.5 & $m_{t r}, \mathrm{~kg}$ & 45.4 \\
\hline$k_{t f}, \mathrm{~N} \cdot \mathrm{m}^{-1}$ & 192000 & $k_{t r}, \mathrm{~N} \cdot \mathrm{m}^{-1}$ & 192000 \\
\hline$k_{s f}, \mathrm{~N} \cdot \mathrm{m}^{-1}$ & 17000 & $k_{s r}, \mathrm{~N} \cdot \mathrm{m}^{-1}$ & 22000 \\
\hline$c_{s f}, \mathrm{~N} \cdot s \mathrm{~m}^{-1}$ & 1500 & $c_{s}, \mathrm{~N} \cdot s \mathrm{~m}^{-1}$ & 1500 \\
\hline$c_{t f}, \mathrm{~N} \cdot s \mathrm{~m}^{-1}$ & 100 & $c_{t r}, \mathrm{~N} \cdot s \mathrm{~m}^{-1}$ & 100 \\
\hline$a, \mathrm{~m}$ & 1.3 & $b, \mathrm{~m}$ & 1.5 \\
\hline
\end{tabular}

According to the control model of time-delayed vibration reduction control shown in Fig. 1, the coordinate origin is selected at the respective equilibrium positions of the wheel and the vehicle body, and Newton's second law is used to write the dynamic motion equation of the body suspension system:

$$
\left.\begin{array}{l}
m_{t f} \ddot{x}_{t f}+c_{s f}\left(\dot{x}_{t f}-\dot{x}_{s f}\right)+k_{s f}\left(x_{t f}-x_{s f}\right)+c_{t f}\left(\dot{x}_{t f}-\dot{x}_{q f}\right)+k_{t f}\left(x_{t f}-x_{q f}\right)-u_{t f}=0 \\
m_{t r} \ddot{x}_{t r}+c_{s r}\left(\dot{x}_{t r}-\dot{x}_{s r}\right)+k_{s r}\left(x_{t r}-x_{s r}\right)+c_{t r}\left(\dot{x}_{t r}-\dot{x}_{q r}\right)+k_{t r}\left(x_{t r}-x_{q r}\right)-u_{t r}=0 \\
m_{c} \ddot{x}_{c}+c_{s f}\left(\dot{x}_{s f}-\dot{x}_{t f}\right)+k_{s f}\left(x_{s f}-x_{t f}\right)+c_{s r}\left(\dot{x}_{s r}-\dot{x}_{t r}\right)+k_{s r}\left(x_{s r}-x_{t r}\right)+u_{t f}+u_{t r}=0 \\
I_{c} \ddot{\phi}_{c}-a c_{s f}\left(\dot{x}_{s f}-\dot{x}_{t f}\right)-a k_{s f}\left(x_{s f}-x_{t f}\right)+b c_{s r}\left(\dot{x}_{s r}-\dot{x}_{t r}\right)+b k_{s r}\left(x_{s r}-x_{t r}\right)-a u_{t f}+b u_{t r}=0
\end{array}\right\}
$$


When the body pitch angle $\varphi_{c}$ is small, the expression of the vertical displacement at the end point of each sprung mass of the body suspension is approximately (2):

$$
\left.\begin{array}{l}
x_{s f}=x_{c}-a \phi_{c} \\
x_{s r}=x_{c}+b \phi_{c}
\end{array}\right\} \text {. }
$$

tion:

Eq. (1) is simplified and identified by matrix equa-

$$
M \ddot{X}+\mathrm{C} \dot{X}+K X+P \mathrm{U}=K_{t} X_{q}+\mathrm{C}_{t} \dot{X}_{q},
$$

where:

$$
\begin{aligned}
& M=\left[\begin{array}{cccc}
m_{t f} & 0 & 0 & 0 \\
0 & m_{t r} & 0 & 0 \\
0 & 0 & m_{c} & 0 \\
0 & 0 & 0 & I_{c}
\end{array}\right], X=\left\{\begin{array}{c}
x_{t f} \\
x_{t r} \\
x_{c} \\
\phi_{c}
\end{array}\right\}, U=\left\{\begin{array}{c}
u_{t f} \\
u_{t r} \\
0 \\
0
\end{array}\right\}, X_{q}=\left\{\begin{array}{c}
x_{q f} \\
x_{q r} \\
0 \\
0
\end{array}\right\}, \\
& K=\left[\begin{array}{cccc}
k_{s f}+k_{t f} & 0 & -k_{s f} & a k_{s f} \\
0 & k_{s r}+k_{t r} & -k_{s r} & -b k_{s r} \\
-k_{s f} & -k_{s r} & k_{s f}+k_{s r} & -a k_{s f}+b k_{s r} \\
a k_{s f} & -b k_{s r} & -a k_{s f}+b k_{s r} & a^{2} k_{s f}+b^{2} k_{s r}
\end{array}\right] \text {, } \\
& P=\left[\begin{array}{cccc}
-1 & 0 & 0 & 0 \\
0 & -1 & 0 & 0 \\
1 & 1 & 0 & 0 \\
-a & b & 0 & 0
\end{array}\right], K_{t}=\left[\begin{array}{cc}
k_{t f} & 0 \\
0 & k_{t r} \\
0 & 0 \\
0 & 0
\end{array}\right], C_{t}=\left[\begin{array}{cc}
c_{t f} & 0 \\
0 & c_{t r} \\
0 & 0 \\
0 & 0
\end{array}\right]
\end{aligned}
$$

\section{Research on vibration reduction strategy of vehicle active suspension system}

\subsection{Selection of time-delayed feedback control strategy}

As shown in Fig. 1, the time-delayed feedback object of $u_{t f}(t)$ and $u_{t r}(t)$ is body or wheel. However, in practical applications, due to the flexibility of sensor selection, the type of time-delayed feedback (time-delayed feedback control signal) mainly includes the single time-delayed feedback type of displacement [13], velocity [14] and acceleration [15] and the combination of them [16]. As the time-delayed stability and time-domain response characteristics of $1 / 4$ vehicle active suspension system based on time-delayed feedback control in the effective frequency band are demonstrated, if the vehicle body is taken as the object of timedelayed feedback, under the optimal time-delayed feedback control, the performance evaluation indexes of vehicle active suspension system can be improved to varying degrees based on the time-delayed feedback control strategies such as body displacement or velocity. However, in the effective frequency band of vehicle vibration caused by road roughness, there are system stability switching and instability phenomena. If the wheel is taken as the object of time-delayed feedback, under the optimal time-delay inverse control, the active suspension system of $1 / 4$ vehicle based on the timedelayed feedback control of wheel displacement is stable in the effective frequency band of vehicle vibration caused by road roughness, and the ride comfort and handling stability of the vehicle are significantly improved under random road excitation.

\subsection{Selection of design variables}

To take the control force $u_{t f}(t)$ and $u_{t r}(t)$ with timedelayed feedback based on vertical displacement of front and rear wheels as an example, the expression of time domain function is shown in (4):

$$
\left.\begin{array}{l}
u_{t f}(t)=g_{t f} \cdot x_{t f}\left(t-\tau_{t f}\right) \\
u_{t r}(t)=g_{t r} \cdot x_{t r}\left(t-\tau_{t r}\right)
\end{array}\right\},
$$

where: $g=\left(g_{t}, g_{t r}\right)$ is the feedback gain and $\tau=\left(\tau_{t f}, \tau_{t r}\right)$ is the time delay. For the time-delayed feedback control forces based on different types of wheel time-delayed feedback, the value of the feedback gain is quite different from that of the time delay. The overall effect of vibration reduction is also different.

\subsection{Frequency domain response transfer function}

Let the root of the characteristic equation of Eq. (3) be $s$, after Laplace transformation, and the result can be written as:

$$
\left(M s^{2}+\mathrm{C} s+K\right) X(s)+P \mathrm{U}(s)=\left[K_{\mathrm{t}}+\mathrm{C}_{\mathrm{t}} s\right] X_{\mathrm{q}}(s) .
$$

Let $P \cdot U(s)=P_{1} \cdot X(s)$, then:

$$
\left(M s^{2}+\mathrm{C} s+K+P_{1}\right) X(s)=\left[K_{\mathrm{t}}+\mathrm{C}_{\mathrm{t}} s\right] X_{\mathrm{q}}(s)
$$

where: $P_{1}=\left[\begin{array}{cccc}-g_{t f} \cdot e^{-s \tau_{t f}} & 0 & 0 & 0 \\ 0 & -g_{t r} \cdot e^{-s \tau_{t r}} & 0 & 0 \\ g_{t f} \cdot e^{-s \tau_{t f}} & g_{t r} \cdot e^{-s \tau_{t r}} & 0 & 0 \\ -a \cdot g_{t f} \cdot e^{-s \tau_{t f}} & b \cdot g_{t r} \cdot e^{-s \tau_{t r}} & 0 & 0\end{array}\right]$.

The transfer function matrix $\mathrm{H}(\mathrm{s})$ can be obtained from Eq. (6):

$$
H(s)=\frac{X(s)}{X_{\mathrm{q}}(s)}=\frac{K_{\mathrm{t}}+\mathrm{C}_{\mathrm{t}} s}{M s^{2}+\mathrm{C} s+K+P_{1}} .
$$

The frequency response transfer function of vehicle vibration can be obtained by taking place of $s$ by $i \omega$, that is:

$$
H(i \omega)=\frac{X(i \omega)}{X_{\mathrm{q}}(i \omega)}=\frac{K_{\mathrm{t}}+\mathrm{C}_{\mathrm{t}}(i \omega)}{-\omega^{2} M+i \omega \mathrm{C}+K+P_{1}} .
$$

\subsection{Selection of optimization objective function}

In view of the fact that the vertical vibration and pitching angle vibration at the center of mass of the vehicle body are important performance evaluation indexes to measure the ride comfort and ride comfort of the vehicle, the minimum dimensionless weighted sum of the amplitude frequency characteristic function values of the vertical acceleration and pitching angular acceleration of the vehicle body is taken as the optimization objective function to measure the vehicle driving characteristics. The optimization of objective function is shown as: 


$$
\begin{aligned}
& \operatorname{MinJ}(g, \tau)=c_{1} \frac{|H(i \omega)|_{\ddot{x}_{c} \sim x_{q}}-\min |H(i \omega)|_{\ddot{x}_{c} \sim x_{q}}}{\max |H(i \omega)|_{\ddot{x}_{c} \sim x_{q}}-\min |H(i \omega)|_{\ddot{x}_{c} \sim x_{q}}}+c_{2} \frac{|H(i \omega)|_{\ddot{\phi}_{c} \sim x_{q}}-\min |H(i \omega)|_{\ddot{\phi}_{c} \sim x_{q}}}{\max |H(i \omega)|_{\ddot{\phi}_{c} \sim x_{q}}-\min |H(i \omega)|_{\ddot{\phi}_{c} \sim x_{q}}} \\
& \text { s.t. }\left\{\begin{array}{l}
\underline{g}_{t f} \leq g_{t f} \leq \bar{g}_{t f} \\
\underline{g}_{t r} \leq g_{t r} \leq \bar{g}_{t r} \\
\underline{\tau}_{t f} \leq \tau_{t f} \leq \bar{\tau}_{t f} \\
\underline{\tau}_{t r} \leq \tau_{t r} \leq \bar{\tau}_{t r}
\end{array}\right\} .
\end{aligned}
$$

3.5. Selection of constraint conditions and weight coefficients

The constraint condition is in the range of feedback gain $g$ and feedback delay $\tau$. Obviously, the selection of time-delayed feedback control parameters should meet the requirements of vehicle ride comfort. The output response of suspension dynamic travel and tire dynamic displacement should also be considered. The numerical search range of the above two kinds of parameters are to be set: the feedback gain $\bar{g}_{t f}$ and $\bar{g}_{t r}$ are 1.5 times of the equivalent stiffness of the front and rear suspension, $\underline{g}_{t f}$ and $\underline{g}_{t r}$ are -0.5 times of the equivalent stiffness of the front and rear suspension. The numerical search range of time delay $\tau=\left(\tau_{t f}, \tau_{t r}\right)$ is assumed to be $0 \leq \tau \leq 0.5$. In expression (9), $c_{1}$ and $c_{2}$ are weight coefficients. The selection of the weight coefficient reflects the importance of the vertical acceleration of the center of mass and the pitching angular acceleration of vehicle body. Equal attention to the optimization is paid to them. The weighting coefficient $c_{1}=c_{2}=0.5$ is taken.

\section{Optimization of feedback control parameters of vehi- cle active suspension system}

\subsection{Time-domain model of road roughness}

In the process of vehicle driving, the feedback signals of vehicle suspension control system are all time domain signals. Establishing a reasonable time-domain model of road roughness is one of the most important issues in the follow-up study of vehicle ride comfort and stability. At present, the main methods to simulate road random excitation model at home and abroad are harmonic superposition method, filtered white noise method, Fourier inverse change method and time series model method. In view of the time delay vibration reduction effect of vehicle body under the condition of road harmonic excitation is remarkable, the time domain model of random road excitation based on harmonic superposition method is selected in this paper. That is, the road input spectral density is converted into a series of discrete sine waves. The random harmonic superposition algorithm is used to sum the sine wave functions corresponding to each time and frequency cell in the effective frequency band and the sum is expressed as trigonometric functions [17-18]. The time domain model of random road roughness as shown in formula (10) can be obtained:

$$
\begin{aligned}
& x_{q}(t)=\sum_{1}^{k} \sqrt{2 G_{q}\left(f_{\text {mid }-j}\right) \cdot \Delta f_{j}} . \\
& \cdot \sin \left(2 \pi f_{\text {mid }-j} t+\theta_{j}\right)(j=1, \cdots k) .
\end{aligned}
$$

As shown in Eq. (10), $\Delta f_{j}$ is the time frequency interval, $G_{q}\left(f_{\text {mid-j }}\right)$ is the road roughness coefficient, $f_{\text {mid-j }}$ is the interval center frequency, $\theta_{j}$ is the random phase angle uniformly distributed in $(0,2 \pi)$.

4.2. Optimization of feedback control parameters of vehicle active suspension system based on time-delayed feedback control

Set the vehicle speed $v=60 \mathrm{~km} / \mathrm{h}$ to drive over the C-level road, the road roughness coefficient is $G_{q}\left(f_{\text {mid-j }}\right)=$ $256 \times 10^{-6} \mathrm{~m}^{3}$. Generally, the frequency range of vehicle vibration caused by road roughness is about $0.25-15 \mathrm{~Hz}$. In this paper, the lower limit and the upper limit of the time frequency of the road acting on the tire excitation are taken as $f_{1}=0.25 \mathrm{~Hz}$ and $f_{2}=0.25 \mathrm{~Hz}$ respectively. According to the product of time frequency and space frequency $f=v \cdot n$, the lower limit of effective spatial frequency $n_{1}=0.015 \mathrm{~m}^{-1}$ and the upper limit of effective spatial frequency $n_{2}=0.9 \mathrm{~m}^{-}$ ${ }^{1}$ of road roughness power spectral density are obtained. In order to avoid frequency aliasing, according to the constraint of sampling interval $\Delta l, \Delta l$ can be selected as 0.015 $\mathrm{m}^{-1}$. The effective spatial frequency interval $\left(n_{1}, n_{2}\right)$ of road roughness power spectral density is divided into $k=59$ small areas, in turn, the center frequency of each space cell is $n_{\text {mid }-j}=0.015 \cdot j+0.0075$ for $j=(1,2, \cdots 59)$. Then the interval length of time frequency is $\Delta f_{j}=0.25$, which leads to $f_{\text {mid }-j}=$ $0.25 \cdot j+0.125$ for $j=(1,2, \cdots 59)$.

Finally, according to the constraint conditions of the time-delayed feedback control parameters and the timedomain model of the random road roughness, the equivalent harmonic excitation method is used to construct the particle swarm optimization algorithm with $J(g, \tau)$ as the fitness function, so as to obtain the optimal value $\left(g_{o p}, \tau_{o p}\right)$ of the feedback control parameters in the effective frequency band. That is, in the effective frequency band $\left(f_{1}, f_{2}\right)$, a series of discrete frequency points are selected at equal intervals and the band is divided into multiple cells. Taking the harmonic excitation at the center frequency $f_{\text {mid }-j}=0.25 \cdot j+0.125$ for $j=(1,2, \cdots 59)$ of each cell as the optimization strategy of the equivalent harmonic excitation of the whole cell, a particle swarm optimization (PSO) algorithm with the minimum dimensionless weighted sum of the amplitude frequency characteristic functions of vertical acceleration and pitching angular acceleration as the optimization objective function is constructed. In the optimization process of timedelayed feedback control parameters, the fixed form of time-delay feedback control parameters (local optimal values) obtained by Fourier transform and frequency domain analysis methods are usually difficult to effectively deal with the characteristics of road random excitation condi- 
tions. In order to further improve the vibration reduction effect of vehicle suspension system under random excitation of road surface, according to the frequency function expression of vertical acceleration and pitching angular acceleration of vehicle body shown in Eq. (11):

$$
\left\{\begin{array}{l}
\left|H\left(i \omega_{\text {mid }-j}\right)\right|_{\ddot{x}_{c} \sim x_{q}}=\omega^{2}\left|\frac{X_{\mathrm{c}}\left(i \omega_{\text {mid }-j}\right)}{X_{\mathrm{q}}\left(i \omega_{\text {mid }-j}\right)}\right| \\
\left|H\left(i \omega_{\text {mid }-j}\right)\right|_{\ddot{\phi}_{c} \sim x_{q}}=\omega^{2}\left|\frac{\psi_{\mathrm{c}}\left(i \omega_{\text {mid-j }}\right)}{X_{\mathrm{q}}\left(i \omega_{\text {mid }-j}\right)}\right|
\end{array} .\right.
$$

the amplitude $\left|x_{q}\left(f_{\text {mid-j }}\right)\right|$ of the road excitation at the center frequency $f_{\text {mid-j } j}=0.25 \cdot j+0.125$ for $j=(1,2, \cdots 59)$ of each interval is assumed to be the peak value of the road excitation in the effective frequency band $\left(f_{1}, f_{2}\right)$, which can effectively reduce the amplitude of vertical acceleration based on the center frequency of each interval in the whole frequency band. Therefore, on the basis of this optimization strategy, 200 particles are randomly selected for iterative optimization, the optimal value of the time-delayed feedback control parameters of the vehicle active suspension system in the effective frequency band is obtained, i.e., $\left(g_{o p}, \tau_{o p}\right)=$ $=(-5552.3 \mathrm{~N} / \mathrm{m} 22017 \mathrm{~N} / \mathrm{m} 0.2591 \mathrm{~s} 0.0001 \mathrm{~s})$ and the amplitude frequency characteristic curve of the vertical acceleration of the vehicle body in the effective frequency band as shown in Fig. 2 are obtained.

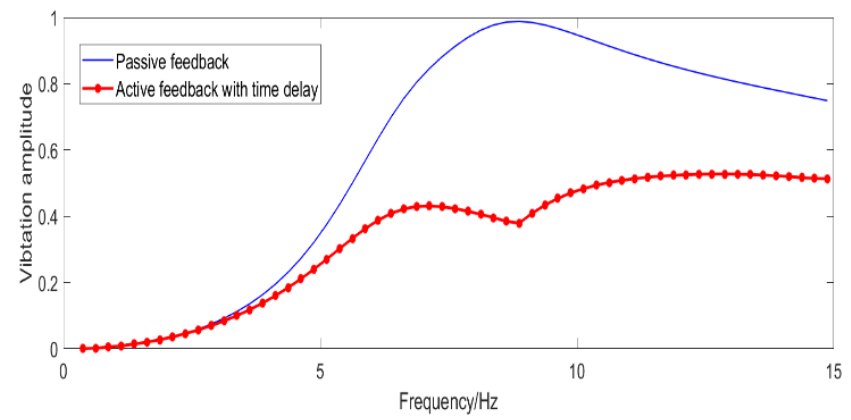

Fig. 2 The amplitude frequency characteristic curves of the fitness function in the effective frequency band

The frequency domain simulation results shown in Fig. 2 show that compared with the passive control suspension system, the vehicle vibration amplitude of active suspension system based on time-delayed feedback control with wheel displacement is significantly reduced under the optimal time-delayed feedback control. The corresponding amplitudes at $f=8.8750 \mathrm{~Hz}$ and $f=12.8750 \mathrm{~Hz}$ are reduced from 0.9883 and 0.8139 in passive control to 0.3794 and 0.5273 in delayed feedback control respectively. 0.9883 is the peak value of the passive suspension system before optimization in the whole frequency band and 0.5273 is the peak value of the optimized suspension system with timedelayed feedback control in the whole frequency band. In addition, the amplitude frequency response curve of the vehicle active suspension system based on the time-delayed feedback control with the vertical displacement of wheel changes relatively gently in the effective frequency band. The vibration reduction effect of vehicle body is remarkable. Therefore, the vibration isolation performance of vehicle suspension system is significantly improved under the optimal time-delayed feedback control.

\section{Stability analysis of time-delayed feedback control for vehicle active suspension system}

After the introduction of time-delayed feedback control, the feedback gain and feedback delay will affect the stability of the system. In order to ensure that the vehicle active suspension system with time-delayed feedback control can work in a stable state, it is necessary to analyze its stability. In this paper, the frequency-domain scanning method is applied to analyze the stability of vehicle active suspension system based on time-delayed feedback control of wheel displacement, so as to obtain the corresponding virtual root and time-delay value [19]. Then the complete stable interval of the system can be obtained. The analysis process is as follows:

Firstly, the characteristic equation of vehicle active suspension system based on time-delayed feedback control with the vertical displacement of wheel can be obtained from Eq. (6) as follows:

$$
\operatorname{det}\left(M s^{2}+\mathrm{C} s+K+P_{1}\right)=0
$$

Secondly, the feedback control parameters of the vehicle active suspension system are successively substituted into the characteristic equation (12), and the relationship diagrams of $|z|$ and $\omega$ as shown in Fig. 3 can be obtained.

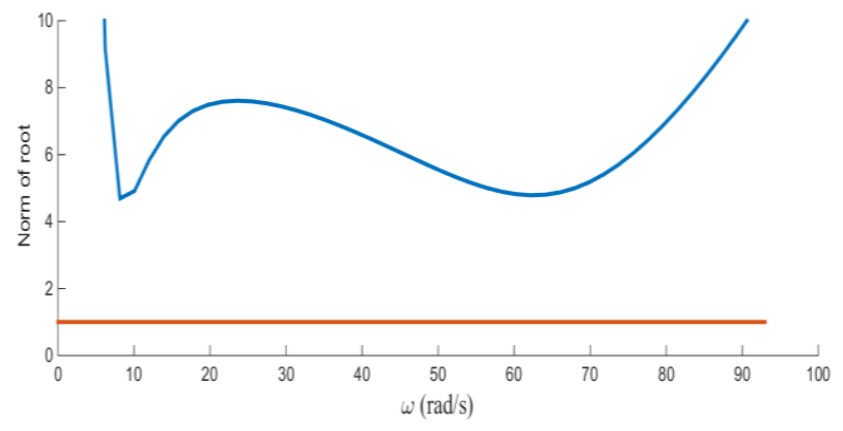

a) The module of $z_{1}$

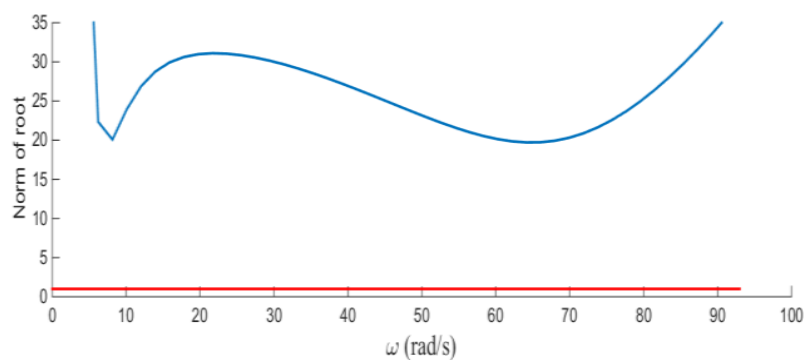

b) The module of $z_{2}$

Fig. 3 The modules of $z$ in vehicle active suspension system based on time-delayed feedback control of wheel displacement

Finally, the crossover frequency $\omega$ and the corresponding time delay are calculated. It can be seen from the diagram of $|z|$ and $\omega$ shown in Fig. 3 that the real root has no cross frequency in the vehicle active suspension system based on time-delayed feedback control of wheel displacement, it means that the whole system has no ride through frequency. Therefore, from the stability analysis of the above vehicle active suspension system, it can be seen that the vehicle active suspension system with time-delayed 
feedback control of wheel displacement is stable in the effective frequency band.

\section{Time-domain simulation analysis and verification of road surface under random excitation}

In order to verify the correctness of the theoretical results, it is necessary to conduct numerical simulation analysis and verification of the time-domain response of the performance evaluation index of the vehicle active suspension system under road excitation according to the optimal value of the time-delayed feedback control parameters. With the C-level road roughness as the excitation and the help of Matlab platform, according to the optimal value of time-delayed feedback control parameters, the performance evaluation indexes of vehicle active suspension system based on time-delayed feedback control of wheel displacement, the simulation results of vehicle body vertical acceleration, pitch acceleration, suspension dynamic stroke, tire dynamic displacement and other performance evaluation indexes are compared and analyzed. The time-domain simulation results are shown in Fig. 4.

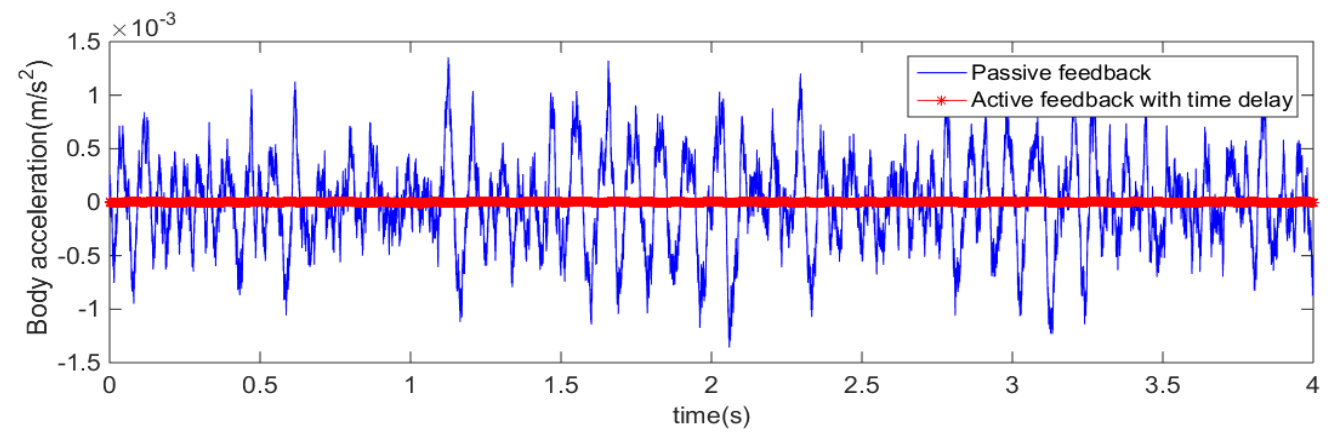

a) Vertical acceleration of vehicle body

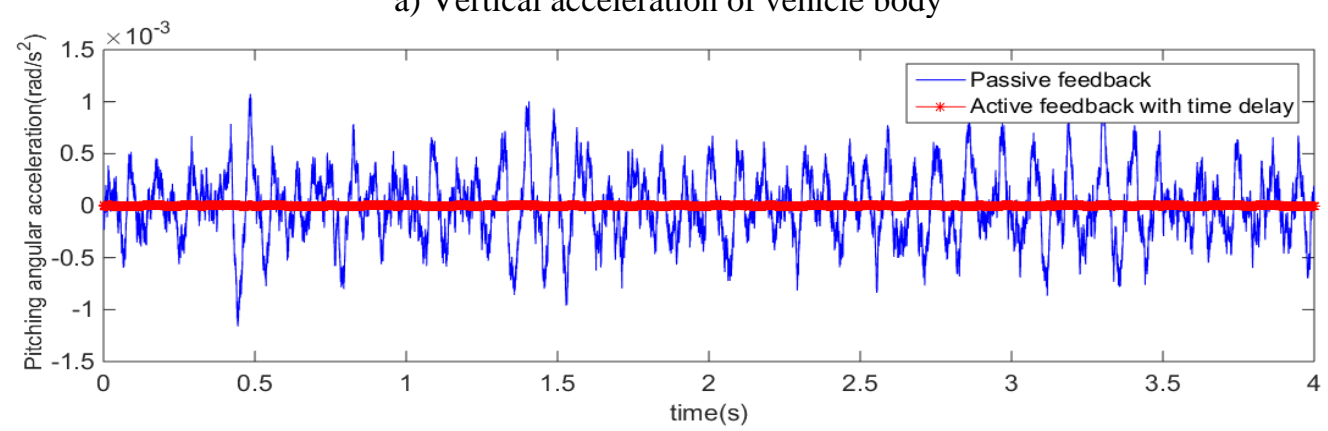

b) Pitching angular acceleration of vehicle body

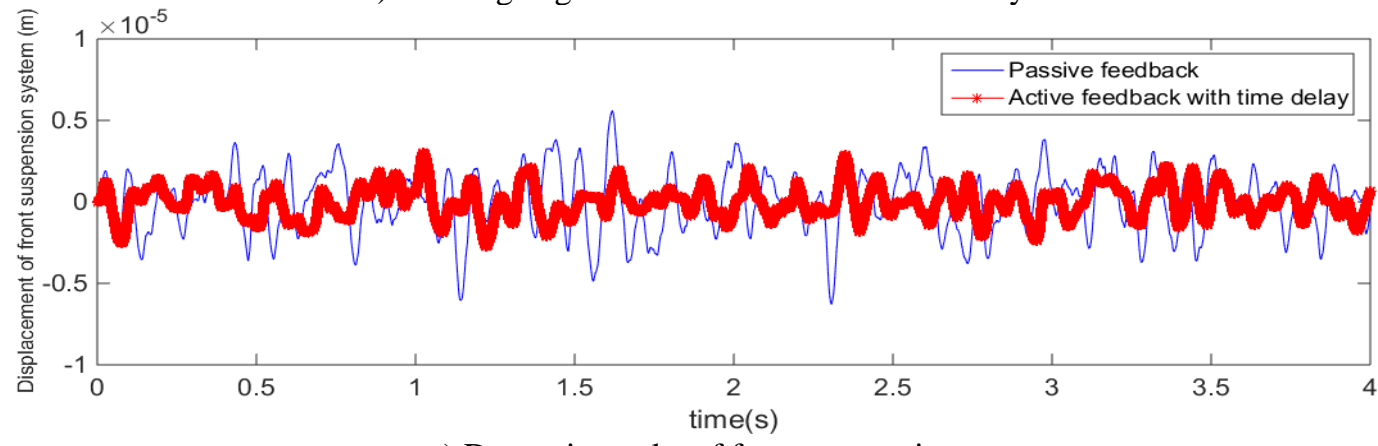

c) Dynamic stroke of front suspension

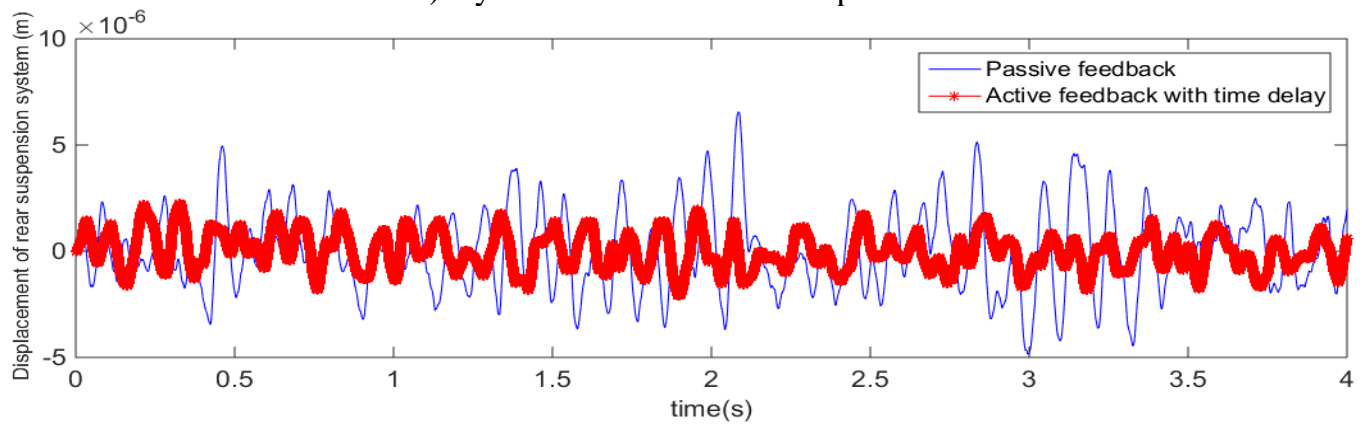

d) Dynamic stroke of rear suspension

Fig. 4Time-domain simulation results of various performance evaluation indexes of vehicle suspension system 


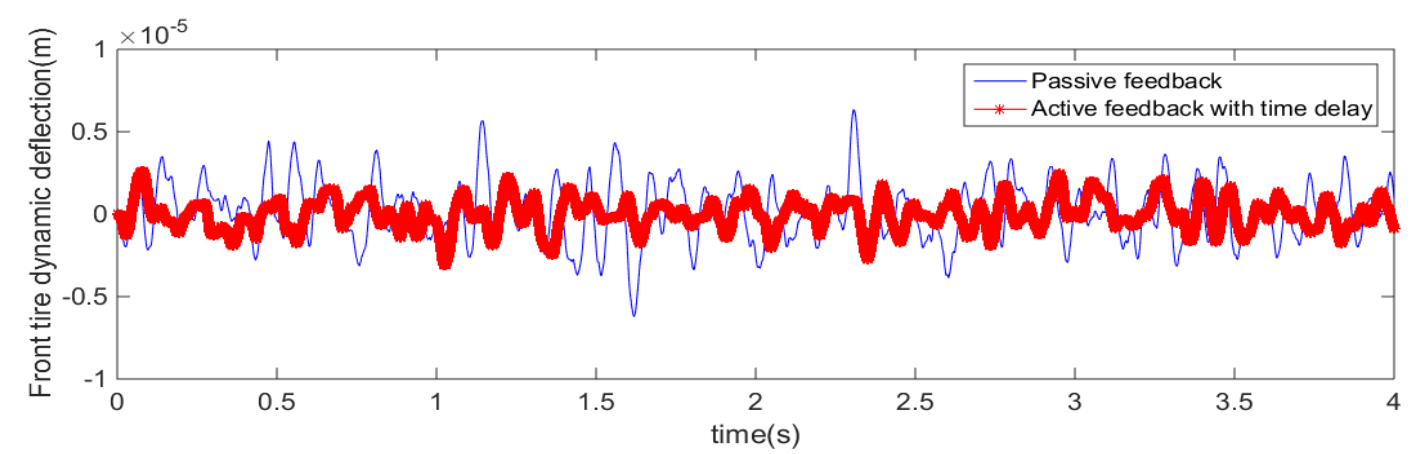

e) Front tire movement displacement

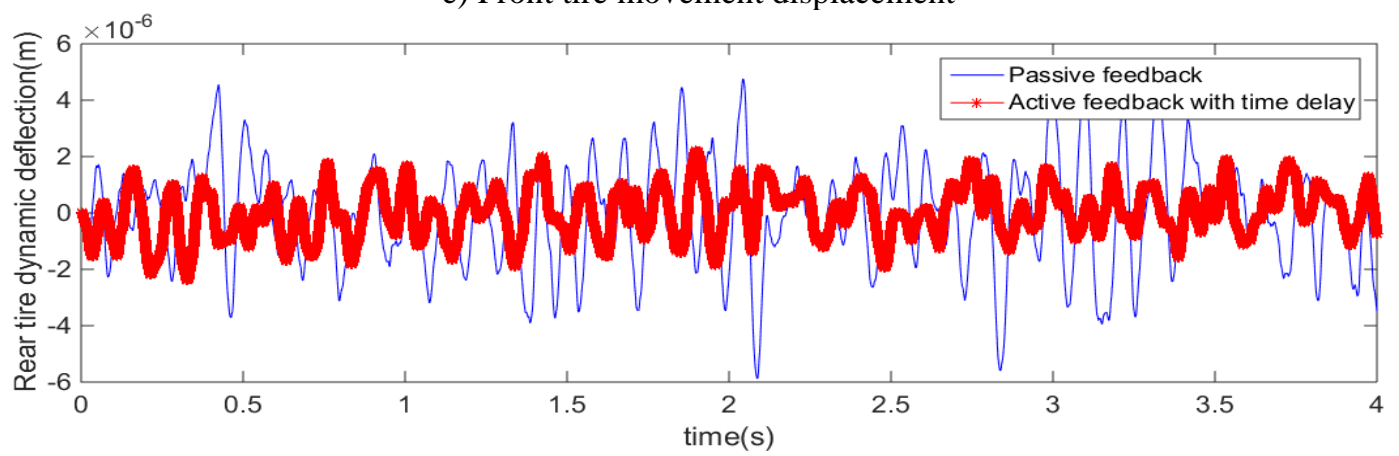

f) Rear tire movement displacement

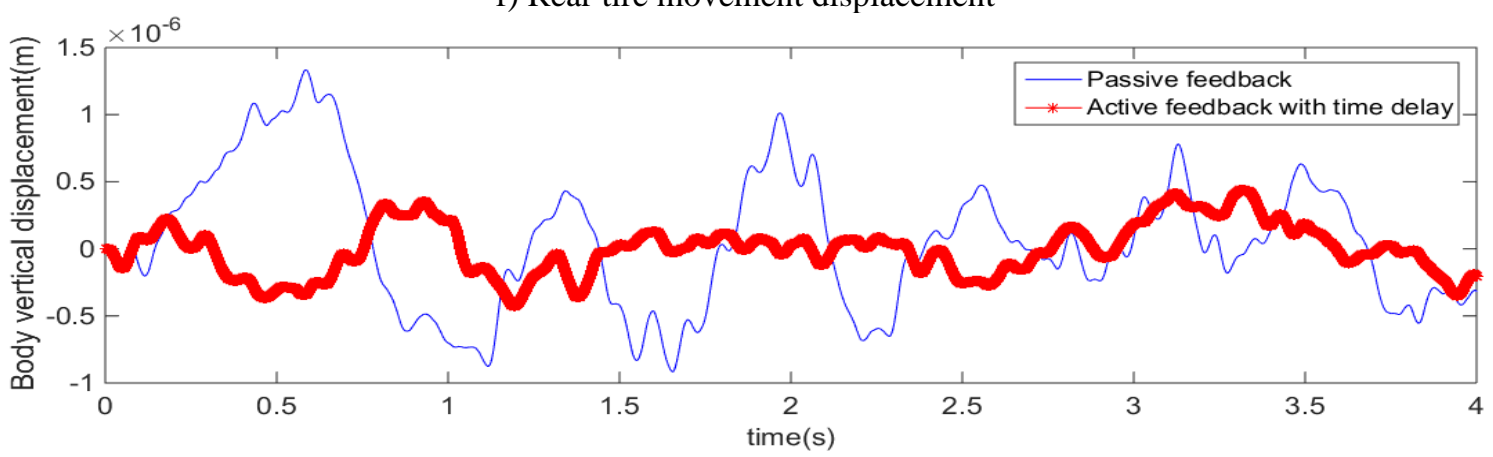

g) Vertical displacement of vehicle body

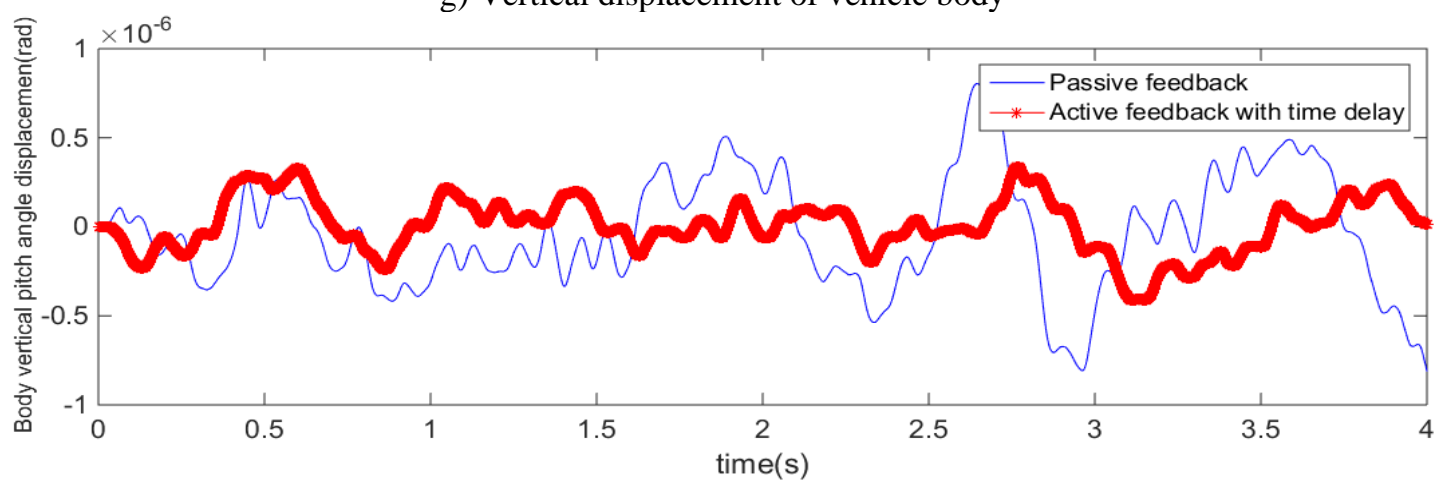

h) Pitching angular displacement of vehicle body

Fig. Continuation

\subsection{Results and discussion}

According to the comparison of root mean square values of performance evaluation indexes of vehicle passive suspension system under random road excitation and vehicle active suspension system with time-delayed feedback control as shown in Table 2, the following conclusions can be drawn:

1) Compared with the passive control, the time-delayed feedback control significantly reduces the amplitude of vertical acceleration and pitching angular acceleration at the body mass center. The corresponding root mean square values are reduced from $0.41183 \mathrm{~mm} / \mathrm{s}^{2}, 0.00032 \mathrm{rad} / \mathrm{s}^{2}$ in passive control to $0.0031511 \mathrm{~mm} / \mathrm{s}^{2}, 0.0000025 \mathrm{rad} / \mathrm{s}^{2}$ in time-delayed feedback control. So the optimization efficiency is $99.2439 \%$ and $99.2053 \%$ respectively.

2) Compared with the passive control, the time-delayed feedback control reduces the amplitude of the front and rear suspension's dynamic travel significantly. The corresponding root mean square values are reduced from 1.8859 um, 1.9468 um in passive control to $1.02456 \mathrm{um}$, 0.89616 um in time-delayed feedback control. So the optimization efficiency is $45.6735 \%$ and $53.9667 \%$ respectively. 
3) Compared with the passive control, the time-delayed feedback control significantly reduces the amplitude of the front and rear tire movement displacement. The corresponding root mean square values are reduced from $1.8283 \mathrm{um}, 1.8707 \mathrm{um}$ in passive control to $0.98189 \mathrm{um}$, $0.91653 \mathrm{um}$ in time-delayed feedback control. So the optimization efficiency is $46.2939 \%$ and $51.0067 \%$ respectively.
4) Compared with the passive control, the amplitude of vertical displacement and pitching angular displacement of vehicle body are significantly reduced by time-delayed feedback control. The corresponding root mean square values are reduced from $0.50885 \mathrm{um}, 0.32547 \mathrm{um}$ in passive control to $0.19115 \mathrm{um}, 0.15421 \mathrm{um}$ in time-delayed feedback control. So the optimization efficiency is $62.4348 \%$ and $52.6182 \%$ respectively.

Table 2

Comparison of root mean square values of performance evaluation indexes of passive suspension and active suspension with time-delayed feedback control under random excitation

\begin{tabular}{|l|c|c|c|}
\hline Performance evaluation index & Passive suspension & Time-delayed feedback suspension & Rate of change \\
\hline Vertical acceleration, $\mathrm{mm} / \mathrm{s}^{2}$ & 0.41183 & 0.0031511 & $99.2349 \%$ \\
\hline Body pitch angular acceleration, $\mathrm{rad} / \mathrm{s}^{2}$ & 0.00032 & 0.0000025 & $99.2053 \%$ \\
\hline Dynamic stroke of front suspension, um & 1.88590 & 1.02456 & $45.6735 \%$ \\
\hline Dynamic stroke of rear suspension, um & 1.94680 & 0.89616 & $53.9667 \%$ \\
\hline Front wheel movement displacement, um & 1.82830 & 0.98189 & $46.2939 \%$ \\
\hline Rear tire movement displacement, um & 1.87070 & 0.91653 & $51.0067 \%$ \\
\hline Vertical displacement of vehicle body, um & 0.50885 & 0.19115 & $62.4348 \%$ \\
\hline Pitching angular displacement of vehicle body, um & 0.32547 & 0.15421 & $52.6182 \%$ \\
\hline
\end{tabular}

\section{Conclusions}

In this paper, the mechanical model of four-degreeof-freedom vehicle active suspension system based on timedelayed feedback control with vertical displacement of wheel is studied, the optimal value of the time-delayed feedback control parameters of vehicle suspension system in effective band is obtained by using the optimization strategy based on "equivalent harmonic excitation" and particle swarm optimization algorithm. Through the optimization design of time-delayed feedback control parameters and the simulation analysis of time-domain response of performance evaluation indexes of vehicle active suspension system, the following conclusions can be obtained:

1) Compared with the passive suspension system, the vehicle active suspension system based on time-delayed feedback control with vertical displacement of wheel under the optimal time-delayed feedback control can effectively broadens the vibration absorption bandwidth of the vehicle suspension system.

2) Compared with the passive suspension system, the vehicle active suspension system based on the time-delayed feedback control with vertical displacement of wheel significantly improves the ride comfort and handling stability of the vehicle under the disturbance of road random excitation. Thus, the comprehensive performance of the suspension is improved.

3) The validity and feasibility of the time-delayed feedback control optimization strategy based on "equivalent harmonic excitation" are verified, which provides a theoretical reference for the optimization design of time-delayed feedback control parameters of vehicle suspension system.

\section{Acknowledgement}

The author(s) disclosed receipt of the following financial support for the research, authorship, and/or publication of this article: This study was supported by National Natural Science Foundation of China (Grant No. 51275280) and Shandong Province Natural Science Foundation of China (Grant No. ZR2015EL048).

\section{References}

1. Wang, R. C.; Chen, L.; Zhang, X. L.; Zhu, X. H. 2012. Design and test of semi-active air suspension system of vehicle, Transactions of the Chinese Society for Agricultural Machinery 43(4): 6-9. http://dx.doi.org/10.6041/j.issn.1000-1298.2012.04.00.

2. Mihai, I.; Andronic, F. 2014. Behavior of a semi-active suspension system versus a passive suspension system on an uneven road surface, Mechanika 20(1): 64-69. http://dx.doi.org/10.5755/j01.mech.20.1.6591.

3. Zhao, C. H.; Chen, S. A.; Wang, J. H. 2015. Influences of stiffness and damping parameters on control of active suspension based on LQG, Transactions of the Chinese Society for Agricultural Machinery 46(12): 301-308, 354. http://dx.doi.org/10.6041/j.issn.10001298.2015.12.041.

4. Song, G.; Xu, C. C. 2013. Stochastic optimal preview control of active vehicle suspension with time-delay consideration, Transactions of the Chinese Society for Agricultural Machinery 44(6): 1-7. https://doi.org/ 10.6041/j.issn.1000-1298.2013.06.001.

5. Kou, F. R.; Fan, Y. Q.; Zhang, C. W.; Du, Q. F. 2016. Time delay compensation control of semi-active suspension with vehicle electro-hydrostatic actuator, China Mechanical Engineering 27(15): 2111-2117. http://dx.org/10.3969/j.issn.1004-132X.2016.15.022

6. Yan, G.; Fang, M. X.; Dong, Q. F.; Ji, R. J. 2018. Time-delayed feedback control of vehicle suspension system based on state Transformation, Transactions of the Chinese Society for Agricultural Machinery 34(10): 54-61. http://dx.org/10.11975/j.issn.1002-6819.2018.10.006.

7. Xu, J.; Chung, K. W.; Zhao, Y. Y. 2010. Delayed saturation controller for vibration suppression in a stainless-steel beam, Nonlinear Dynamics 62(1-2): 177-193. http://dx.doi.org/10.1007/s11071-010-9708-4.

8. Sun, Y. X.; Xu, J. 2015. Experiments and analysis for a controlled mechanical absorber considering delay effect, Journal of Sound \& Vibration 339: 25-37. 
https://doi.org/ 10.1016/j.jsv.2014.11.005.

9. Zhao, Y. Y.; Xu, J. 2006. Delayed resonator and its effects on vibrations in primary system, Journal of Vibration Engineering 19(4): 548-552.

https://doi.org/10.1007/s11390-006-0261-z.

10. Olgac, N.; Holm-Hansen, B. T. 1994. A novel active vibration absorption technique: delayed resonator, Journal of Sound \& Vibration 176(1): 93-104. https://doi.org/10.1006/jsvi.1994.1360.

11. Du, H. P.; Zhang, Nong. 2007. Control of active vehicle suspensions with actuator time delay, Journal of Sound \& Vibration 301(1-2):236-252. https://doi.org/10.1016/j.jsv.2006.09.022.

12. Liu, J. J.; Sun, Y. X.; Li, S. 2020. Parameter optimization of time-delayed feedback control for vehicle suspension system in finite frequency band, Journal of Mechanical \& Electrical Engineering 37(1):54-58. https://doi.org/10.3969/j.issn.1001-4551.2020.01.010.

13. El-Ganaini, W. A.; Kandil, A.; Eissa, M.; Kamel, M. 2014. Effects of delayed time active controller on the vibration of a nonlinear magnetic levitation system to multi excitations, Journal of Vibration and Control 22(5): 1257-1275. https://doi.org/ 10.1177/1077546314536753.

14. Xu, J.; Chen, Y.; Chung, K. W. 2015. An improved time-delay saturation controller for suppression of nonlinear beam vibration, Nonlinear Dynamics 82(4): 16911707. https://doi.org/ 10.1007/s11071-015-2270-3.

15. An, F.; Li, J.; Zhang, W. L. 2015. Advances and review on the research of the time-delayed acceleration feedback control. Chinese Journal of Applied Mechanics 32(6): 985-991(in Chinese). https://doi.org/ 10.11776/cjam.32.06.C027.

16. Eissa, M.; Kandil, A.; El-Ganaini, W. A.; Kamel, M. 2015. Analysis of a nonlinear magnetic levitation system vibrations controlled by a time-delayed proportional-derivative controller, Nonlinear Dynamics 79(2): 12171233. https://doi.org/ 10.1007/s11071-014-1738-X.

17. Au, F. T. K.; Cheng, Y. S.; Cheung, Y. K. 2001. Effects of random road surface roughness and long-term deflection of prestressed concrete girder and cablestayed bridges on impact due to moving vehicles, Comput Struct 79(8): 853-872.
https://doi.org/10.1016/S0045-7949(00)00180-2.

18. Schiehlen, W. Hu. B. 2003. Spectral simulation and shock absorber identification, International Journal of Non-Linear Mechanics 38(2): 161-171. https://doi.org/10.1016/S0020-7462(01)00053-1.

19. Barkin, A. I. 2006. Stability of linear time-delay systems, Automation and Remote Control 67(3): 345-349. https://doi.org/10.1134/S0005117906030015.

\section{Y. Guo, C. Ren}

\section{RESEARCH ON VIBRATION REDUCTION OF HALF- VEHICLE ACTIVE SUSPENSION SYSTEM BASED ON TIME-DELAYED FEEDBACK CONTROL WITH WHEEL DISPLACEMENT}

S u m m a r y

In this paper, a strategy of time-delayed feedback control based on wheel displacement and an optimization method of time-delayed feedback control parameters based on "equivalent harmonic excitation" are proposed for vehicle active suspension system with time-delayed feedback control. In this way, the optimal values of time-delayed feedback control parameters of the vehicle suspension system in the effective frequency band are obtained. Finally, through the stability analysis of suspension system and the numerical simulation analysis of the time-domain response of the suspension system performance evaluation index, the effectiveness of the time-delayed feedback control strategy based on the wheel displacement and the superiority of the optimization method based on the "equivalent harmonic excitation" are verified. Therefore, it provides a theoretical basis for the selection of time-delayed feedback control strategy and the optimal design of time-delayed feedback control parameters of vehicle active suspension system.

Keywords: time-delayed feedback control, equivalent harmonic excitation, active suspension, optimal values, stability.

Received April 02, 2021

Accepted February 04, 2022 